\title{
Osteochondral lesions of the talus: are we ready for metal?
}

\author{
Riccardo D’Ambrosi, Federico Giuseppe Usuelli \\ IRCCS Istituto Ortopedico Galeazzi, Unità Operativa C.A.S.C.O, Milan, Italy \\ Correspondence to: Riccardo D’Ambrosi, MD. IRCCS Istituto Ortopedico Galeazzi, Unità Operativa C.A.S.C.O, Milan, Italy. \\ Email: riccardo.dambrosi@hotmail.it. \\ Provenance: This is an invited Editorial commissioned by Section Editor Jian Xu (Orthopaedic Surgery and Sports Medicine, The First Affiliated \\ Hospital of Shenzhen University, Shenzhen, China). \\ Comment on: Vuurberg G, Reilingh ML, van Bergen CJA, et al. Metal Resurfacing Inlay Implant for Osteochondral Talar Defects After Failed \\ Previous Surgery: A Midterm Prospective Follow-up Study. Am J Sports Med 2018;46:1685-92.
}

Submitted Aug 25, 2018. Accepted for publication Sep 10, 2018.

doi: 10.21037/atm.2018.09.24

View this article at: http://dx.doi.org/10.21037/atm.2018.09.24

The treatment for osteochondral lesions of the talus (OLTs) presents several strategies based on the type and degree of the lesion. The main factors to evaluate are the age of the patient, functional demands, the size and the location of the defect, and any instability or deformity of the ankle. Biological repair techniques, such as autologous chondrocyte transplantation (ACI), autologous matrix-induced chondrogenesis (AMIC), osteochondral transplantation and bone marrow stimulation (BMS) are aimed at treating symptomatic isolated localized cartilage defects in younger patients without ankle deformity. However, many doubts persist regarding the treatment in middle-aged patients, affected by isolated symptomatic talar defect: these patients are neither fit for regenerative medicine, nor for resurfacing or replacement techniques (1).

In recent years, we have observed a "back to the future" due to the use of a surgical strategy that involves focal talus resurfacing metal implants (2). The concept of resurfacing was born in the 1960s with Sir John Charnley, while the first reference as regards foot and ankle is dated 1981 with Daniels et al. $(3,4)$. The ratio for resurfacing is to recreate ankle anatomy based on intraoperative topographic mapping. The stability of the implant is ensured by virtue of the inset position relative to the surrounding articular cartilage. Moreover, this technique allows concomitant surgical soft tissue and bony procedures because it doesn't increase joint volume. Metal resurfacing can be considered a minimally invasive surgery, with reduced surgical time, simple and cannulated implantation technique, and scarce blood loss. The procedure has been widely accepted for the shoulder and hip with typically near-complete unipolar articular coverage (5).

In the ankle the main indication is for the symptomatic middle-aged (40-55 years) active patient where biological or surgical treatments have failed or are ineffective.

This method can be regarded as the final attempt at joint preservation and occasionally the indication may be enlarged in primary OLTs, in particular when the lesion is incipient or the patient is too young for ankle replacement or arthrodesis.

In order to obtain a stable and effective implant articulating against the opposing tibial cartilage, three factors must be evaluated: the implant must bond to the host bone; the surrounding cartilage should not be damaged but rather adhere to the implant; and finally, it is mandatory that the opposing cartilage withstands the new biomaterial over time (6).

Despite the numerous current techniques described in the literature, to date there is still no treatment that can be defined the gold standard or superior to another, neither for primary or secondary OLTs $(7,8)$.

In this scenario the biological techniques are gaining more and more popularity and attention, with the aim of enhancing the local biological environment; plateletrich plasma (PRP) is the autologous plasma fraction of the blood containing concentrated platelets and, in most cases, white blood cells, providing a natural conductive scaffold and containing many growth factors. PRP can be used as a conservative treatment, or in association with surgical procedure with the aim to augment the healing potential of the cartilage. Although most clinical trials report promising outcomes, a degree of ambivalence towards PRP remains. 
This is due to the heterogeneity of studies involving PRP that use different preparation. Another issue concerns whether PRP should be activated or not (1).

Bone marrow aspirate concentrate (BMAC) is the result of different density gradient centrifugation of bone marrow aspirated from the iliac crest containing platelets and growth factors, typically in lower concentrations than PRP. Evidence supporting the use of BMAC is increasing, although literature is still scarce (9).

Finally, as regards regenerative medicine, the focus is shifting to adipose-derived stem cells (ADSCs). All this interest is mainly due to their high number in the human body (ADSCs are $5 \%$ of nucleated cells in adipose tissue), the simplicity of harvesting, and their rapid expansion and high proliferative potential. These mesenchymal stem cells can differentiate into different cellular lines such as chondrocytes, osteoblasts, tenocytes and muscle cells both in vitro and in vivo (10).

Despite the fact that clinical results related to biological treatments are promising, there are still some doubts regarding their use: in fact, there is not always uniformity in the method of collection and preparation, and in many cases clinical results do not correlate with radiological ones, in which the lesion shows no noticeable improvements.

However, surgical techniques are separated into reparative, restorative and regenerative. Reparative strategies aim to stimulate tissue repair through debridement and microperforations at the level of the lesion. In reconstructive techniques the damaged cartilage is replaced with chondral or osteochondral grafts, while regenerative techniques exploit the ability of cells to differentiate and replicate to reconstruct injured tissues (1).

BMS is a reparative procedure indicated in patients with smaller lesions up to $1.5 \mathrm{~cm}^{2}$. Despite the successful outcomes, neo-formed cartilage presents inferior mechanical properties, such as stiffness and resilience, and therefore presents a high risk of deterioration (1).

Autologous osteochondral transplantation (AOT) involves the replacement of injured cartilage by implanting a cylindrical osteochondral graft harvested from a nonweight bearing portion of the knee into the talar lesion. While clinical studies report good to excellent outcomes without deterioration over time, issued have been raised regarding poor integration of the graft surface with the native bone and cartilage, cyst formation at the level of the implant, and deterioration of the graft (1).

ACI technique was initially conceived and experimented in a rabbit and only subsequently has acquired clinical use in cartilage repairs. Despite good early clinical results, the main issue of the technique remains the donor site morbidity (1).

Marix-induced autologous chondrocyte implantation (MACI) is a modification of ACI where chondrocytes are embedded into a matrix scaffold, which is then fixed to the articular surface using fibrin glue. The use of a matrix scaffold, which has the advantage of retaining the chondrocytes in the defect has gained some traction and demonstrated promising results (1).

AMIC technique was described for the first time by Benthien and Behrens, introducing the concept of clot stabilization following microfracture through use of a type I/III collagen scaffold (11). Recently the technique has been described entirely arthroscopically (AT-AMIC) with proposed advantages of smaller incisions, reduced morbidity and less soft-tissue dissection (12).

Matrix-associated stem cell transplantation (MAST) is a single stage recent modification of AMIC with a potentially higher concentration of stem cells in the implanted matrix (1).

Metal resurfacing has found much of its clinical use in revision surgery: recently Vuurberg et al. (2) analyzed 38 patients who underwent metal resurfacing after previous failed OLTs surgery. Mean age at surgery was 39 years and patients were evaluated after a mean follow-up of 5.1 years. Two patients underwent subsequent revision surgery by means of an ankle arthrodesis. Significant clinical improvement was noted in all scores apart from pain at rest, the Foot and Ankle Outcome Score (FAOS) symptoms subscale, and the 36-Item Short Form Health Survey (SF-36) mental component scale; $77 \%$ of the patients resumed physical activity after a mean of 4.1 months. In consideration of the results reported, authors sustained that metal resurfacing is an effective technique at mid-term for OLTs after failed previous surgery.

Despite these promising results, many issues remain related to this type of treatment: first of all, although the HemiCAP offers 15 different offset sized based on articular surface of the talus, it is a challenge to reconstruct ankle anatomy: in fact, the talus presents an enormous variability which is accentuated in patients with ankle osteoarthritis that show a flattening and a more pronounced frontal aspect of the talus $(13,14)$. Could a custom-made system be the future?

Another important aspect in HemiCAP system concerns the positioning, in fact as for the AOT, implantation accuracy plays a key-role (15). A biomechanical study highlighted that metal resurfacing is able to recover more than $90 \%$ of the contact area. If the implant protrudes by 
Table 1 Algorithm treatment for primary osteochondral talar defects

\begin{tabular}{lll}
\hline Age (years) & Lesion size $\left(\mathrm{cm}^{2}\right)$ & Treatment \\
\hline$<55$ & $<1.5$ & $\begin{array}{l}\text { Bone marrow stimulation }+ \\
\text { adipose-derived stem cells }\end{array}$ \\
& $>1.5$ & $\begin{array}{l}\text { Arthroscopic autologous } \\
\text { matrix-induced } \\
\text { chondrogenesis }\end{array}$ \\
& & Metal resurfacing \\
\hline
\end{tabular}

Table 2 Algorithm treatment for osteochondral talar defects after previous failed surgery

\begin{tabular}{lll}
\hline Age (years) & Lesion size & Treatment \\
\hline$<55$ & Any & Metal resurfacing \\
$\geq 55$ & Any & Total ankle replacement \\
\hline
\end{tabular}

$0.25 \mathrm{~mm}$, peak contact stress increases by $220 \%$. In case the implant recessed $0.25 \mathrm{~mm}$, the peak values of implant-oncartilage contact stress decreased, while there is an increase in peak values of the cartilage-on-cartilage contact area. Focal implant resurfacing substantially restored kinematics but did not restore the stresses to the levels in the intact specimens (16). It would be very interesting to analyze whether in Vuurberg's study there is a possible correlation between failures and implant placement, particularly for patients who subsequently underwent an arthrodesis or those who reported rest pain (2).

Furthermore, future biomechanical studies should evaluate the osteoinductive and osteointegrative capabilities of these implants to understand the stability over time.

Moreover it is important to note the high rate of re-intervention to remove the screws from the medial malleolus presented in the study; Leumann et al. suggest that optimal location for medial malleolar osteotomy is at the medial ankle edge (17). Clinical results using this location showed low mid-term morbidity and only little long-term morbidity, but hardware removal is a common procedure with significant costs $(17,18)$.

Undoubtedly planning medial malleolus osteotomy in the right location would help to reduce the risk of consistent articular damage, but it is still the main issue. With our AT-AMIC studies, we proved that the use of a proper tool (Hinterman speader with $2.5 \mathrm{k}$-wire) allows good exposure to over $2 / 3$ of all talar domes lesions, with the exception of the far posterior ones $(12,19)$.

We recommend considering this technique even for metal HemiCAP. Good planning is needed in order to avoid unnecessary osteotomies.

Furthermore, we advocate considering lateral approach and fibula osteotomy instead of medial malleolus osteotomy. It has been recently stated as a safe and reliable approach for total ankle replacement. Several advantages may be linked to a similar procedure: reduction of the articular damage, chance to easily address lateral instability and varus ankle deformity (shortening fibula procedure) which are both often related to osteochondral lesions (20).

Vuurberg's article presents interesting thoughts as regards metal resurfacing indications': in fact the authors reported that in 3 patients the HemiCAP system was used as first implant, while 20 patients had undergone previous surgery more than once (2). In this respect there is still a lot of confusion about surgical indications of hemiCAP, especially considering the excellent results of the ankle replacement; two patients of the study in which the metal resurfacing has failed, have been subjected to a consequent arthrodesis, with all the disadvantages related to this type of surgery (20).

In these two patients the authors sustain that ankle arthrodesis was the only possible therapeutic option after metal resurfacing failure due to the large taper interlock (10.3-17.4 mm) and concomitant bone loss, excluding savage procedures such as resurfacing total ankle replacement $(21,22)$.

In these situations it would be interesting to evaluate if the replacement surgery is more difficult than a first implant, analyzing the surgical times and the patient's blood loss.

A last consideration on this technique concerns the risk of infection; in the article no patients reported postoperative infections, but being a metal implant the risk exists, leaving a number of open questions: How to treat these cases? Remove everything? Perform an arthroscopic debridement? Implant a spacer? Treat only with antibiotics?

In light of the fact that there is still no gold standard in the literature and relying on our experience we have created an algorithm treatment for osteochondral lesions, both primary and secondary. In case of primary lesions in young patients the first choice should be biological/regenerative, also linked to the size of the lesion, while metal resurfacing should be considered only in patients with symptomatic osteochondral lesion over the age of 55 years (Table 1).

In case of revision, metal resurfacing should be considered in young patients $(<55)$, while in older patients total ankle replacement is the treatment of choice (Table 2). 


\section{Acknowledgements}

None.

\section{Footnote}

Conflicts of Interest: Dr. Usuelli reports personal fees from Integra and Geistlich, grants and personal fees from Zimmer, outside the submitted work. Dr. D'Ambrosi has no conflicts of interest to declare.

\section{References}

1. McGoldrick NP, Murphy EP, Kearns SR. Osteochondral lesions of the ankle: The current evidence supporting scaffold-based techniques and biological adjuncts. Foot Ankle Surg 2018;24:86-91.

2. Vuurberg G, Reilingh ML, van Bergen CJA, et al. Metal Resurfacing Inlay Implant for Osteochondral Talar Defects After Failed Previous Surgery: A Midterm Prospective Follow-up Study. Am J Sports Med 2018;46:1685-92.

3. Charnley J. Using Teflon in arthroplasty of the hip-joint. J Bone Joint Surg Am 1966;48:819.

4. Daniels AU, Samuelson KM, Rusin KA. Talonavicular joint surface anatomy and prototype resurfacing prostheses. Foot Ankle 1981;2:5-14.

5. Ettinger S, Stukenborg-Colsman C, Waizy H, et al. Results of HemiCAP® Implantation as a Salvage Procedure for Osteochondral Lesions of the Talus. J Foot Ankle Surg. 2017;56:788-92.

6. Martinez-Carranza N, Ryd L, Hultenby K, et al. Treatment of full thickness focal cartilage lesions with a metallic resurfacing implant in a sheep animal model, 1 year evaluation. Osteoarthritis Cartilage 2016;24:484-93.

7. Dahmen J, Lambers KTA, Reilingh ML, et al. No superior treatment for primary osteochondral defects of the talus. Knee Surg Sports Traumatol Arthrosc 2018;26:2142-57.

8. Lambers KTA, Dahmen J, Reilingh ML, et al. No superior surgical treatment for secondary osteochondral defects of the talus. Knee Surg Sports Traumatol Arthrosc 2018;26:2158-70.

9. Yasui Y, Wollstein A, Murawski CD, et al. Operative Treatment for Osteochondral Lesions of the Talus: Biologics and Scaffold-Based Therapy. Cartilage 2017;8:42-9.

10. D'Ambrosi R, Indino C, Maccario C, et al. Autologous Microfractured and Purified Adipose Tissue for
Arthroscopic Management of Osteochondral Lesions of the Talus. J Vis Exp 2018;(131).

11. Benthien JP, Behrens P. Autologous Matrix-Induced Chondrogenesis (AMIC): Combining Microfracturing and a Collagen I/III Matrix for Articular Cartilage Resurfacing. Cartilage 2010;1:65-8.

12. Usuelli FG, D'Ambrosi R, Maccario C, et al. Allarthroscopic AMIC® (AT-AMIC®) technique with autologous bone graft for talar osteochondral defects: clinical and radiological results. Knee Surg Sports Traumatol Arthrosc 2018;26:875-81.

13. Wiewiorski M, Hoechel S, Anderson AE, et al. Computed Tomographic Evaluation of Joint Geometry in Patients With End-Stage Ankle Osteoarthritis. Foot Ankle Int 2016;37:644-51.

14. Siegler S, Toy J, Seale D, et al. The Clinical Biomechanics Award 2013 -- presented by the International Society of Biomechanics: new observations on the morphology of the talar dome and its relationship to ankle kinematics. Clin Biomech (Bristol, Avon) 2014;29:1-6.

15. Latt LD, Glisson RR, Montijo HE, et al. Effect of graft height mismatch on contact pressures with osteochondral grafting of the talus. Am J Sports Med 2011;39:2662-9.

16. Anderson DD, Tochigi Y, Rudert MJ, et al. Effect of implantation accuracy on ankle contact mechanics with a metallic focal resurfacing implant. J Bone Joint Surg Am 2010;92:1490-500.

17. Leumann A, Horisberger M, Buettner O, et al. Medial malleolar osteotomy for the treatment of talar osteochondral lesions: anatomical and morbidity considerations. Knee Surg Sports Traumatol Arthrosc 2016;24:2133-9.

18. Fenlon C, Murphy EP, Galbraith JG, et al. The burden of hardware removal in ankle fractures: How common is it, why do we do it and what is the cost? A ten-year review. Foot Ankle Surg 2018. [Epub ahead of print].

19. Usuelli FG, de Girolamo L, Grassi M, et al. AllArthroscopic Autologous Matrix-Induced Chondrogenesis for the Treatment of Osteochondral Lesions of the Talus. Arthrosc Tech 2015;4:e255-9.

20. Usuelli FG, D'Ambrosi R, Manzi L, et al. Treatment of Ankle Osteoarthritis with Total Ankle Replacement Through a Lateral Transfibular Approach. J Vis Exp 2018;(131).

21. Preis M, Bailey T, Marchand LS, et al. Can a Three- 
Component Prosthesis be Used for Conversion of Painful Ankle Arthrodesis to Total Ankle Replacement? Clin Orthop Relat Res 2017;475:2283-94.

22. Preis M, Bailey T, Marchand LS, et al. Conversion

Cite this article as: D'Ambrosi R, Usuelli FG. Osteochondral lesions of the talus: are we ready for metal? Ann Transl Med 2018;6(Suppl 1):S19. doi: 10.21037/atm.2018.09.24 of painful tibiotalocalcaneal arthrodesis to total ankle replacement using a 3-component mobile bearing prosthesis. Foot Ankle Surg 2017. [Epub ahead of print]. 